\title{
CAPITAL SOCIAL E DESEMPENHO PERCEBIDO EM MICRO E PEQUENAS EMPRESAS
}

\section{SOCIAL CAPITAL AND PERCEIVED PERFORMANCE IN MICRO AND SMALL ENTERPRISES}

\section{D0I: http://dx.doi.org/10.21714/raunp.v9i1.1514}

\section{Thiago Martins Costa}

Mestre em Administração. Fucape Business School. E-mail: pesquisa@fucape.br

\section{Bruno Felix Von Borell de Araujo}

Doutor em Administração de Empresas. Fucape Business School

E-mail: bfelix@fucape.br

\section{Aziz Xavier Beiruth}

Doutor em Controladoria e Contabilidade. Fucape Business School

E-mail: aziz@fucape.br

Envio em: Junho de 2016

Aceite em: Março de 2017

\section{RESUMO}

Este trabalho propõe a avaliar a relação existente entre três dimensões do Capital Social (su-porte organizacional, institucional e familiar e social) e 0 Desempenho Percebido nas micro e pequenas empresas (MPE). 0 alicerce teórico dessa pesquisa usa a Teoria das Redes Sociais, mas principalmente a Teoria do Capital Social. A amostra nessa pesquisa foi composta por 448 empreendedores, de micro e pequenas empresas brasileiras que foram atendidos pelo SE-BRAE. Com os dados levantados na pesquisa foi utilizada a modelagem de equações estrutu-rais para avaliação das relações hipotetizadas. 0 questionário usado com os empreendedores contém perguntas que permitem captar as percepções destes quanto às dimensões de desem-penho da MPE, e 0 quanto 0 Capital Social pode influenciar o desempenho, sendo esse tam-bém 0 objetivo central dessa pesquisa. Os resultados, de forma geral, colaboram com a literatu-ra no sentido de apresentar uma mensuração robusta e multidimensional do Capital Social e sua relação com o Desempenho Percebido pelas MPEs. Eles apontam um baixo, porém signifi-cante potencial de explicação do desempenho pelo Capital Social, especialmente pelo Suporte Governamental.

Palavras-chave: Capital social. Desempenho Percebido. Micro e pequena empresa. Redes sociais.

\section{ABSTRACT}

This study aims to evaluate the relationship between the three dimensions of Social Capital (organizational, institutional and familiar and social) and the Perceived Performance in micro and small enterprises (MSEs). The theoretical foundation of this research uses the theory of social networks, but mainly the Theory of Social Capital. The sample in this study consisted of 448 entrepreneurs, micro and small Brazilian companies that were attended by SEBRAE. With the data collected in the survey was used structural equation modeling to assess the hy-pothesized relationships. The questionnaire used with entrepreneurs contains questions that allow capturing the perceptions of these dimensions as the performance of the MSEs, and how much capital can influence performance, and this is also the main objective of this research. The results, in general, work with literature in order to provide a robust, multidimensional measurement of Social Capital and its relationship with Performance Perceived by MSEs. They point to a low, but significant performance potential explanation of the capital, especially the Governmental Support.

Keywords: Social Capital. Measurement. Micro and small enterprises. Social Networks. 


\section{INTRODUÇÃO}

As micro e pequenas empresas (MPEs) são componentes importantes e estratégicos para o desenvolvimento econômico mundial, especialmente em países emergentes, como é o caso do Brasil, porque são geradoras importantes de emprego, geram também riqueza nacional e contribuem significantemente para o PIB nesses países (FILION, 1999; PICHARDO; GAR-ZA, 2011 , no prelo; ZAHRA, et al., 2009).

As MPEs compõem a base da pirâmide econômica de um país (PRAHALAD; HART, 2002); entre suas principais características comuns estão o baixo investimento de capital no início de suas atividades, alta taxa de natalidade e de mortalidade, usam mão de obra familiar, a pouca ou nenhuma distinção entre os bens do proprietário e da própria empresa, além de pos-suírem controles inadequados de suas atividades (PICHARDO; GARZA, 2011) impossibili-tando assim que esses empreendedores possam mensurar o desempenho adequadamente em seu empreendimento, e com isso, não compondo estrategicamente uma vantagem competitiva em relação a concorrência (PETARAF; BARNEY, 2003).

Com o objetivo de melhor explicar o desempenho das MPEs, autores têm buscado com-preender os fatores que influenciam o desempenho desse tipo de empresa, mesmo que ainda não haja um consenso na literatura para composição de indicadores financeiros e não-financeiros visando medir e avaliar o desempenho empresarial (MACAU; BRITO, 2010), em especial nas MPEs, tendo em vista não serem precisas em conceder informações corretas sobre seu desempenho (COVIN; SLEVIN, 1989).

Diversos autores têm estudado fatores influenciadores do desempenho nas MPEs. São avaliados antecedentes como as competências empreendedoras, financeiras e econômicas (GILL, 2011), comportamento do empreendedor (MACIEL; CAMARGO, 2010), a cultura organizacional (MOHAMAD et al., 2011), a inovação (TERZIOVSK, 2010), orientação estra-tégica, como por exemplo, recursos humanos, mercado, aprendizagem, e o próprio cliente (BROCKMAN et al., 2012), o planejamento estratégico (LI et al., 2008), e em relação a dife-rentes tipos de suporte, como o apoio familiar, social, institucional e governamental (GILL et al., 2012).

Uma forma de maximizar e potencializar o desempenho nas MPEs é investir no Capital Social mediante as redes sociais, nas quais, pela cooperação, o conhecimento e a informação são compartilhados. Com isso, diminuem as incertezas, a assimetria de informação, cria-se uma vantagem competitiva e aumenta-se a competitividade das empresas participantes e a eficiên-cia coletiva (PROVAN; SYDOW, 2008; UZZI; GILLESPIE, 2002; MESQUITA; LAZZA-RINI, 2008).

Justamente por isso as MPEs têm sido vistas como um campo promissor para a pesquisa de Capital Social (GOLDMARK, 2001), assim como para a redução da pobreza (WOOL-COCK, 2001), especialmente nas economias de países emergentes (TEECE, 2000).

O Capital Social tem sido apontado como uma força motriz por trás do desempenho empresarial, entretanto, poucos estudiosos têm se empenhado em pesquisar esse fenômeno nas MPEs, sobretudo em culturas emergentes (WESTLUND; ADAM, 2010). Um desses estudos foi realizado no México por Pichardo e Garza (2011), que buscam entender o efeito do Capital Social no desempenho das MPEs. Entretanto, pouco se conhece acerca dos efeitos e dos resul-tados desses estudos em outros países emergentes; dessa forma, para fins de comparação entre países na mesma condição, os autores sugerem que o estudo seja realizado em outros países com igual classificação, como, por exemplo, o Brasil.

Assim, a pergunta que pauta a realização dessa pesquisa é: Qual é a relação entre o Capital Social e o Desempenho Percebido nas MPEs?

O objetivo geral deste estudo é avaliar a relação entre o Capital Social composto pelo Suporte Governamental, institucional, familiar e social no Desempenho Percebido das micro e pequenas empresas. Como objetivos específicos, é proposto estimar a relação entre os cons-tructos do Capital Social e o Desempenho Percebido nas MPEs e, também, avaliar das propri-edades psicométricas dos instrumentos utilizados.

Uma vez entendendo a importância das redes sociais para o desempenho das micro e pequenas empresas - inseridas em países emergentes, compostos em sua maioria por ambientes institucionais fracos, 
e com pouca infraestrutura (WRIGHT et al., 2005), é justificável aplicar uma pesquisa no Brasil, a fim de contribuir no avanço dos estudos em termos de conhecimen-to, visando entender como a eficiência coletiva, por intermédio do Capital Social, efetivamen-te contribui no desempenho das micro e pequenas empresas.

Este estudo inicia-se com uma breve introdução sobre o tema; em seguida, apresentamos o referencial teórico no qual mostramos as principais vertentes sobre o tema. Após isso, apre-sentamos as hipóteses, metodologia e instrumento de pesquisa utilizado, e finalizamos com a apresentação das análises e discussões dos resultados encontrados, além das conclusões deste estudo.

\section{REFERENCIAL}

\section{TEÓRICOREFERENCIAL TEÓRICO}

\subsection{MICRO E PEQUENA EMPRESA (MPE)}

As micro e pequenas empresas (MPEs) desempenham um importante papel nas economi-as emergentes. Geram riqueza nacional, criam emprego, fomentam o comércio internacional (LI et al., 2008), contribuem significativamente com a constituição do PIB (FILION, 1999), são uma importante fonte de emprego e vitalidade econômica em países de economia emer-gente (PICHARDO et al., 2011; ZAHRA et al., 2009), além de serem os motores do cresci-mento econômico, são consideradas agentes importantes para o desenvolvimento social e polí-tico de uma comunidade (FITZGERALD et al., 2010).

A definição de micro e pequena empresa possui certa relatividade, e isso significa dizer, que em diferentes países, há distintos critérios para classificar uma organização como micro e pequena (FILION, 1991). Dessa forma, o mais comum, de certa forma para definir MPE, é considerar a evolução econômica através de seu volume de negocio gerado anualmente. Assim no Brasil a Lei complementar $n^{0} 123$, de 14 de dezembro de 2006 define como microempresa a empresa individual de responsabilidade limitada a empresa que gere uma receita bruta anual de até $\mathrm{R} \$$ $360.000,00$, e empresa de pequeno porte aquele que possui, entre $\mathrm{R} \$ 360.000,01$ e $\mathrm{R} \$ 3.600 .000,00$.

Já as características de uma MPE são comuns e constantes na maioria dos países. Pode-mos entender as principais características de uma MPE como empresas de baixa intensidade de capital, altas taxas de natalidade e de mortalidade, forte presença dos sócios-proprietários, familiares como força de trabalho barata nos negócios, poder de decisão concentrado, vínculo estreito entre os proprietários e as empresas, não se distinguindo, principalmente em termos contábeis e financeiros, a pessoa física da jurídica; registros contábeis inadequados; faz seleção e contrato direto com a mão-de-obra; utilização de mão-de-obra não qualificada ou semiqua-lificada; baixo investimento em inovação tecnológica; maior dificuldade de acesso ao financi-amento de capital de giro; e relacionamento de dependência com as empresas de grande porte (PICHARDO et al., 2011).

Ao fazermos um comparativo, na Europa e nos Estados Unidos, de forma geral, o per-centual de participação das MPEs no PIB é relevante e significativo, principalmente quando comparamos ao do Brasil, mesmo que em relação ao total da participação esses números sejam equivalentes, por exemplo, na Espanha, as MPEs correspondem a 99,5\% dos estabelecimentos formais e a $50,6 \%$ do PIB; já na Grécia, eles alcançam $99,4 \%$ e $55,6 \%$, respectivamente, en-quanto na Itália, os índices são de 99,5\% e 55,6\% (EUROPEAN COMISSION, 2009). Da mesma forma nos Estados Unidos da América, conforme o United State Census (2008), as MPEs representam 99,6\% de todos os empregadores nos Estados Unidos e empregam mais de $50 \%$ do total da força de trabalho dos EUA. Isso demonstra o quão substancial é a importân-cia do impacto desse segmento na vida econômica americana quanto européia.

No Brasil, as MPEs representam 99,1\% dos empreendimentos formais, geram $52,2 \%$ dos empregos com carteira assinada e respondem apenas por $20 \%$ do PIB, assim, podemos ver com mais clarividência o impacto que a geração de empregos nas MPEs causa efetivamente no PIB, ou seja, a suposta baixa produtividade das MPEs impacta na produtividade média da economia brasileira (BRASIL, 2009). Dessa forma, mesmo com muitos empregos gerados pe-las MPEs, esses não refletem de maneira robusta impacto na produtividade no PIB.

\subsection{DESEMPENHO PERCEBIDO NAS MPES:}

$\mathrm{Na}$ literatura científica o número de publicações sobre indicadores de desempenho em empresas é vasto, mesmo assim, os pesquisadores ainda não chegaram a uma visão comum sobre a essência e composição dos indicadores de desempenho, bem como a sua medição e métodos de avaliação de desempenho em- 
presarial, o que torna o tema ainda controverso (KO-TANE; MERLINO, 2012; MACAU; BRITO, 2010), isto porque, não há uma única aborda-gem quanto à identificação, classificação, medição e avaliação de indicadores financeiros e não financeiros nas empresas (WIER et al., 2007), e mensurar o desempenho organizacional é estratégico para o empreendedor compor sua vantagem competitiva e sustentável em relação aos seus eventuais concorrentes (PETARAF; BARNEY, 2003).

Dessa forma, uma saída comumente utilizada para esse problema é utilizar escalas do de-sempenho autopercebido, ou seja, os próprios empresários que respondem às pesquisas retra-tam seu desempenho segundo sua percepção. Essa pratica apresenta como viés o fato de que o grau de exigência ou de entendimento dos respondentes quanto ao seu mercado e seu poten-cial desempenho influencia suas respostas, entretanto, isso tem sido comum na literatura sobre o tema, como pode ser notado nos trabalhos de Trigueroi et al., (2012); Engellandt e Riphahn, (2011); Bol e Smith, (2011). Neste trabalho, utilizamos essa última abordagem e, por isso, uti-lizamos o termo "Desempenho Percebido" para o referido constructo.

Para medir o Desempenho Percebido nas MPEs, usaremos as propostas sugeridas por Covin e Slevin (1989), que sugerem, dado a falta de informações financeiras precisas pelas MPEs, e ainda serem relutantes em fornecerem a informação correta e desejada, e que os da-dos financeiros das MPEs não ficam disponíveis, tornando a exatidão deles ainda mais com-plicada, os autores, prevendo a subjetividade nas percepções dos empreendedores em relação ao desempenho financeiro, propõem medir, pela satisfação ou insatisfação, o grau de impor-tância que a empresa percebe a cada um dos seguintes critérios de desempenho financeiro: o nível de vendas, taxa de crescimento, fluxo de caixa, retorno sobre o patrimônio líquido, mar-gem de lucro bruto, o lucro líquido das operações, o lucro em relação às vendas, retorno sobre investimento e capacidade de financiar o crescimento do negócio.

Dadas essas alternativas para a mensuração do desempenho organizacional, diversos es-tudos têm mensurado esse constructo e identificam quais fatores antecedentes melhor o expli-cam. Por exemplo, fatores antecedentes tais como as diferentes fontes de suporte, como o apoio familiar, social, governamental e institucional (GILL et al., 2012), a eficácia do Diretor-Presidente (YANG; YU, 2011), as competências organizacionais empreendedoras, financeiras e econômicas (JÄNKÄLÄ; SILVOLA, 2012), o comportamento empreendedor do fundador (MACIEL; CAMARGO, 2010), os tipos de controle adotados (MACIEL; CAMARGO, 2010) e as características da cultura organizacional (MOHAMAD et al., 2011) têm sido avali-ados como explicadores do desempenho de micro e pequenas empresas.

\subsection{REDES SOCIAIS}

Podemos definir redes sociais como um conjunto de nós ou laços específicos fortes (conexão direta) ou fracos (conexão indireta) (WASSERMAN; FAUST, 1994), que unem pessoas ou empresas em recursos e idéias em prol do interesse coletivo (MARTELETO, 2001). O compartilhamento do conhecimento e as trocas de informações nas redes sociais são reco-nhecidos como uma estratégia, e fonte de vantagem competitiva sustentável, pois potenciali-zam o desempenho nas MPEs participantes de redes sociais (POWELL, 1990, OLK, 2005). Por isso Lin (1999, p. 31), demostra, pelo menos, quatro benefícios diretos que as MPEs po-dem obter através de suas redes sociais: Influência, Credenciais sociais, Suporte ou Apoio, e, Informação.

Devido ao processo de aprendizagem nas Redes Sociais, as MPEs, aumentam a confi-ança coletiva e diminuem as incertezas, consequentemente, aumentam a sua competitividade, legitimação, status, benefícios econômicos e financeiros (UZZI; GILLESPIE, 2002; WU, 2008), isto porque, as redes sociais são boas propagadoras de informações e otimizam o co-nhecimento prático de gestão entre as empresas participantes e, com isso, ampliam o grau de inovação e know how (GELETKANYCZ; HAMBRICK, 1997), impactando, assim, positiva-mente na sobrevivência e no desempenho dentro das empresas (BRASS et al., 2004)

O uso consciente das redes sociais, sob certas condições, pode contribuir para o forta-lecimento de mercados e melhoria do desempenho das empresas, mediante o desenvolvimento da eficiência coletiva (MESQUITA; LAZZARINI, 2008). Isso é possível porquanto é preci-samente pelas redes sociais que as MPEs têm acesso aos recursos necessários para desenvolve-rem vantagens competitivas sustentáveis (SVETLIČIČ et al., 2007).

A condição evolutiva das redes sociais obriga as empresas a atualizarem continuamente suas conexões de negócio (BURT, 2000), assim, nos mercados atuais, as empresas devem es-tabelecer e fortalecer cons- 
tantemente as relações com outras, a fim de se tornarem e permane-cerem competitivas (QUATMAN; CHELLADURI 2008). Por isso, é salutar deduzir ser com-plexo presumir que todas as empresas pertencentes à mesma rede social possuem as mesmas conexões, as mesmas relações, tampouco que essas empresas façam uso delas por igual; assim, não é certo pressupor que todas as empresas pertencentes à mesma rede social obtenham o mesmo nível de benefícios e de desempenho (SYDOW; MILWARD, 2003). Em complemen-to, Zaher e Bell (2005), salientam que uma rede de relacionamento social oferece às empresas participantes o potencial de recursos ofertados pelos membros da mesma rede social, entretan-to cabe a elas ter a expertise e a capacidade de explorá-los.

Quanto maior e melhor forem os tipos de relacionamento existente, dessas empresas nas redes sociais, melhor serão seus acessos a informações e recursos estratégicos de seus par-ceiros, que de outra forma seriam inacessíveis ou muito dispendiosos, dessa forma, as MPEs aproveitam dessas redes sociais visando obter mais recursos, através do Capital Social (MI-KHAILITCHENKO, 2008; NAHAPIET; GOSHAL, 1998).

\subsection{CAPITAL SOCIAL}

Neste estudo, define-se Capital Social como o valor atribuído pelo empreendedor para os benefícios derivados das redes sociais. Esse valor representa a soma do valor real e poten-cial contido e disponível na sua rede social (BURT, 1997).

O Capital Social, por um processo de aprendizagem, enriquece o fluxo de informações nas organizações na exploração de novos mercados (WRIGHT et al., 2005). Diante disso, é estratégico as empresas fazerem a assimilação e o compartilhamento do conhecimento com mais facilidade, formal ou informalmente, por meio de uma rede (TODOROVA; DURISIN, 2007). Devido à natureza intangível e inimitável do Capital Social, esse pode ser considerado uma importante fonte de vantagem competitiva (ZHAO; HSU, 2007). Talvez por isso, a visão dominante na literatura associe de forma positiva as redes sociais e o Capital Social com a ca-pacidade de inovação e desempenho das MPEs (VAN WIJK et al. 2008).

Entretanto, há autores que afirmam serem inconclusivas e controversas as evidências sobre a relação entre o Capital Social e o desempenho nas MPEs como, por exemplo: Rowley e Berman (2000) e Park e Luo (2001) tendo em vista os custos associados com a formação des-tes laços serem elevados e tiram, assim, eventuais investimentos diretos, os quais poderiam ser feitos nas empresas. Outra afirmação destacada pelos autores diz respeito à interconectivida-de, pois quanto maior for, mais complicado é para a empresa obter uma informação estratégica e aproveitá-la em suas operações diárias.

Diversos autores têm estudado os suportes oferecidos às MPEs em diferentes dimen-sões, como por exemplo (GILL et al., 2012). No presente trabalho analisam-se três tipos espe-cíficos de suporte: governamental, institucional, familiar e social.

Por Suporte Governamental entende-se nesse trabalho o apoio derivado do relaciona-mento dos empreendedores gestores das micro e pequenas empresas com funcionários públicos e instituições governamentais. Nesse aspecto é possível incluir, por exemplo, treinamento, ser-viços financeiros e de informação, assistência jurídica e outros benefícios (YIU; LAU, 2008).

Já Suporte Institucional nesse trabalho será entendido como um apoio derivado do re-lacionamento dos empreendedores das micro e pequenas empresas com instituições não gover-namentais: treinamentos, serviços financeiros e de informação, assistência jurídica e outros benefícios dos eventuais apoios derivado do relacionamento dos empreendedores com os fun-cionários dessas instituições (YIU; LAU, 2008).

Suporte Familiar e Social pode ser entendido como apoio prestado pela família, amigos e conhecidos dos empreendedores nas atividades e rotinas do dia a dia, ou apoio financeiro, jurídico, ou ainda, algum tipo de assistência técnica institucional (YIU; LAU, 2008).

Tendo em vista que esses três tipos de suportes podem tanto influenciar quanto serem determinantes no Desempenho Percebido das MPEs, logo estratégicos na criação da vantagem competitiva de uma MPE; portanto, é possível propor hipóteses que permitam testar esse efei-to:

Hipótese 1 - Há uma relação positiva e significante entre o Suporte Governamental re-cebido pela MPE e seu Desempenho Percebido.

Hipótese 2 - Há uma relação positiva e significante entre o Suporte Institucional rece-bido pela MPE e seu Desempenho Percebido.

Hipótese 3 - Há uma relação positiva e significante entre o Suporte Familiar e Social recebido pela MPE e seu Desempenho Percebido.

Dadas as hipóteses apresentadas, é possível criar um modelo estrutural que mostra as relações propostas e hipotetizadas entre os constructos. 


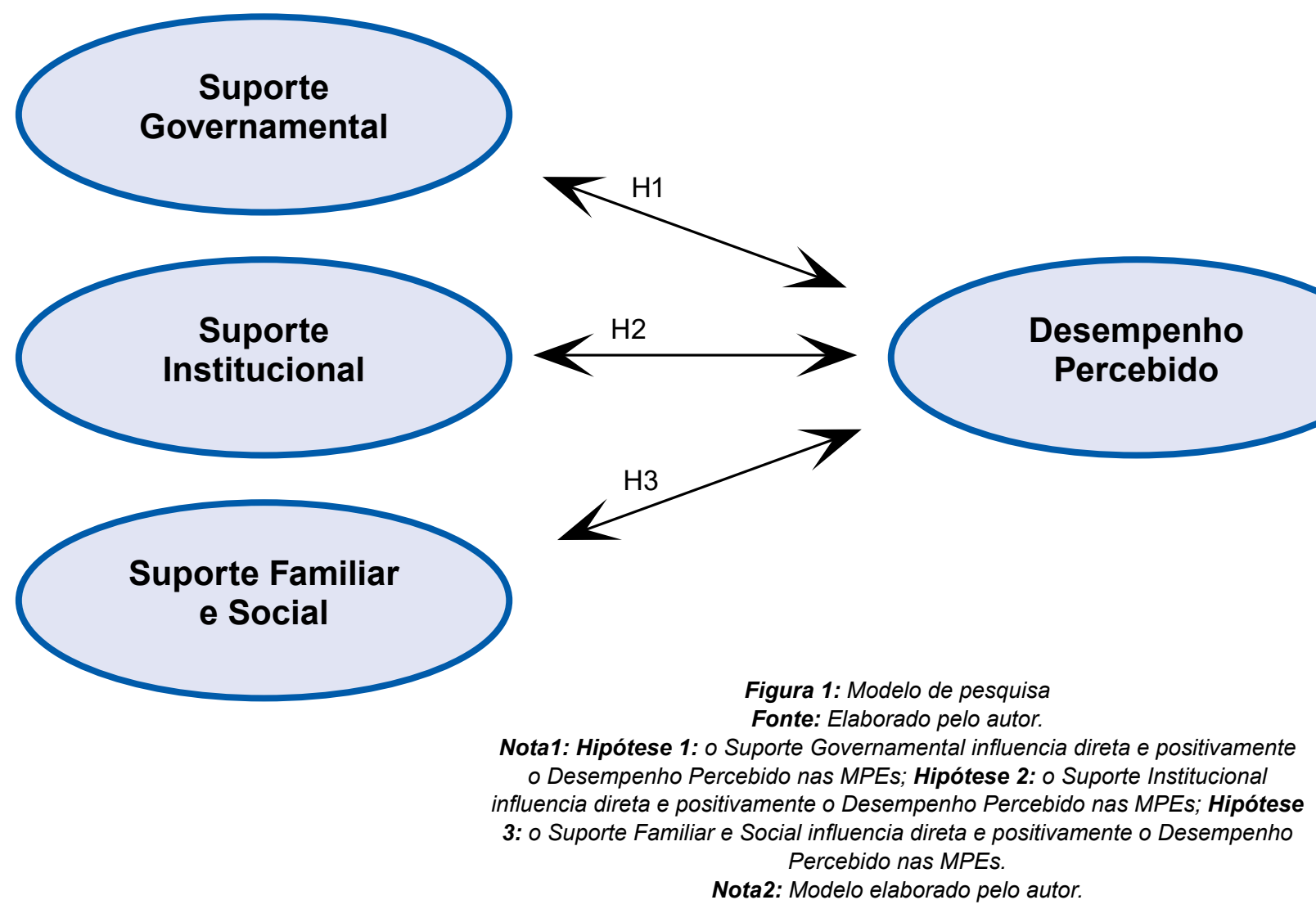

\section{METODOLOGIA}

Para composição da amostra desta pesquisa, usou-se uma base de empreendedores de MPEs, atendidos espontaneamente pelo SEBRAE no Espírito Santo, entre os meses de janeiro de 2009 e dezembro de 2011. Nesse período, foram atendidos 12.768 empresários e candida-tos a empresário. Para delimitar os respondentes, optou-se por convidar para a pesquisa os em-presários que tivessem seus dados completos, obtidos no ato do atendimento, tais como cadas-tro nacional de pessoa jurídica (CNPJ), nome completo, cadastro geral de pessoa física (CPF), meios de comunicação (telefone e e-mail) e faturamento bruto anual declarado igual ou inferi-or a $\mathrm{R} \$ 3.600 .000,00$, de forma a garantir que a amostra fosse confiável e coerente com os propósitos do estudo. Com isso, chegou-se a uma base final válida de 1.127 empreendedores a qual aqui será reconhecida como amostra da pesquisa.

Procedeu-se à aplicação de um questionário fechado, publicado em um site da internet, cujo link foi disponibilizado por e-mail para a amostra da pesquisa. No e-mail convite foi ex-planado tanto o interesse quanto o propósito central do estudo, bem como a necessidade de os respondentes serem os empreendedores responsáveis pela gestão das MPEs

O período de coleta, compreendido entre os meses de janeiro e fevereiro de 2012, foi de 30 dias, com uma comunicação intermediária para estimular os respondentes. Foram coletados 448 questionários respondidos, sem a ocorrência de inválidos ou missing values, o que reflete uma taxa de retorno de aproximada de $39,8 \%$, sendo adequada para os fins do estudo de acordo com a regra empírica de cinco a dez respondentes por assertiva (HAIR JR. et al., 2005). $\mathrm{O}$ instrumento divide-se em quatros blocos: o Bloco I é direcionado ao Desempenho Percebido, composto por 09 variáveis; o Bloco II, direcionado ao Suporte Governamental composto por 09 variáveis; o Bloco III, destinado ao Suporte Institucional também composto por 09 variáveis, e o Bloco IV, destinado ao Suporte Familiar e Social composto por 10 variá-veis, totalizando 37 assertivas.

Inicialmente, segundo o recomendado por Hair Jr. et al. (1998), realizou-se a verificação dos dados da pesquisa em termos de dados ausentes (missing values) e dados atípicos (outli-ers), e nenhum questio- 
nário foi excluído da amostra, fato que pode ser considerado como um indicativo do comprometimento dos respondentes. As questões reversas foram recodificadas. Para a análise dos dados da pesquisa, foi empregada a modelagem de equações estruturais (structural equation modeling - SEM), de acordo com os critérios de Pett et al. (2003), não pressupõem normalidade multivariada da amostra, admitindo também um tamanho pequeno de casos para que se estimem os parâmetros.

Os instrumentos para essa pesquisa originalmente estavam na língua inglesa e, para o perfeito entendimento da pesquisa realizada em território brasileiro, foi feita a tradução reversa do instrumento, conforme proposto por Hudson et al. (2007).

\section{ANÁLISE DOS RESULTADOS}

Os dados demográficos mostram que, a porcentagem de empreendedores do sexo mas-culino participantes da pesquisa (65\%), é quase o dobro do sexo feminino $(35 \%)$. Um pouco mais da metade dos respondentes são do comércio (54\%), seguidos pelos prestadores de servi-ço (29\%) e indústria (15\%). A faixa etária dos respondentes está concentrada entre os 26 e 45 anos (65\%), e o tempo de escolaridade concentra-se entre 11 e 15 anos de estudo (51\%).

Ao analisar o grau de dedicação do empreendedor ao seu empreendimento, notamos que $91 \%$ passam mais de 30 horas por semana se dedicando a ele. Quanto ao numero de pes-soas que se dedicam em tempo integral (8 horas diárias ou mais), 188 (42\%) afirmam ter entre um e cinco colaboradores. Já em relação ao número de pessoas que se dedicam à empresa em tempo parcial (menos de 8 horas diárias) e/ou eventual, 241 (54\%) dos respondentes afirmam também ter entre um e cinco colaboradores.

As características demográficas encontradas neste estudo são semelhantes às relatadas por outros auto- res, como por exemplo, Pichardo e Garza (2011, no prelo $)^{2}$, que fizeram um estudo similar no México, e apresentaram o perfil de seus respondentes como sendo todos empreendedores de MPEs com faturamento anual inferior a U\$20.000, e no máximo com 10 funcionários, sendo que na amostra, somente $47 \%$ dos respondentes mexicanos tinha empre-gados em tempo integral. Dos respondentes, $60 \%$ eram do sexo feminino, 30\% tinham recebi-do uma educação empreendedora formal. A idade média dos empreendedores mexicanos foi de 42 anos, e a média educacional foi de 13 anos de escolaridade, o que equivale naquele país ao primeiro ano do ensino superior. Não houve diferenças significativas na idade ou na educa-ção dos respondentes mexicanos. Em relação ao tipo de negócio dos respondentes, $37 \%$ são do setor industrial, $40 \%$, de serviços e $23 \%$, do comércio.

\subsection{AVALIACCÃO DO MODELO DE MENSURAÇÃO}

Em estudos modelados por equações estruturais (Structural Equation Modeling-SEM), Chin (1998), propõe, para aceitação da validade discriminante, que haja a existência de indi-cadores mais altos nas variáveis latentes que mensuram do que as outras variáveis mensuradas. Uma segunda análise proposta por Chin (1998), e Hair Jr et al. (2005), é a validade convergen-te. Para os autores, a aceitação dessa validade, a variância média extraída (Average Variance Extracted - $A V E$ ) deve ser maior que $50 \%$. A terceira proposta de avaliação da confiabilidade se dá por meio da confiabilidade composta, que deve superar o índice de 0,7 (CHIN, 1998).

A seguir apresentam-se a matriz de cargas cruzadas e uma tabela com a correlação e as estatísticas descritivas dos constructos de $1^{\mathrm{a}}$ ordem, que são usadas como fonte para avaliação das propriedades psicométricas citadas anteriormente. 
TABELA 1: MATRIZ DE CARGAS CRUZADAS

\begin{tabular}{|c|c|c|c|c|c|c|}
\hline $\begin{array}{l}\text { Variáveis } \\
\text { latentes de } \\
1^{\circ} \text { ordem }\end{array}$ & \multicolumn{2}{|c|}{ Indicadores } & 1 & 2 & 3 & 4 \\
\hline \multirow{11}{*}{$\begin{array}{l}\text { 1- Suporte } \\
\text { Familiar e } \\
\text { Social. }\end{array}$} & \multicolumn{6}{|c|}{$\begin{array}{l}\text { O quanto sua empresa recebeu de apoio de seus familiares, amigos e conhecidos, } \\
\text { em cada um dos seguintes aspectos nos últimos três anos? }\end{array}$} \\
\hline & CeFS_1 & Nas atividades cotidianas da sua empresa. & 0,46 & 0,00 & $-0,03$ & 0,11 \\
\hline & CeFS_2 & Vendas. & 0,66 & 0,21 & 0,19 & 0,19 \\
\hline & CeFS_3 & Compras. & 0,56 & 0,21 & 0,21 & 0,12 \\
\hline & CeFS_4 & Serviços financeiros (poupança, crédito, seguros, etc.). & 0,45 & 0,16 & 0,15 & 0,00 \\
\hline & CeFS_5 & Benefícios fiscais. & 0,72 & 0,46 & 0,46 & 0,16 \\
\hline & CeFS_6 & Capacitação técnica. & 0,84 & 0,42 & 0,51 & 0,12 \\
\hline & CeFS_7 & Capacitação gerencial. & 0,84 & 0,44 & 0,49 & 0,15 \\
\hline & CeFS_8 & Serviços de informação. & 0,82 & 0,39 & 0,43 & 0,10 \\
\hline & CeFS_9 & Serviços de contratação de pessoal. & 0,79 & 0,34 & 0,41 & 0,10 \\
\hline & CeFS_10 & Assistência legal. & 0,79 & 0,45 & 0,47 & 0,10 \\
\hline \multirow{10}{*}{$\begin{array}{l}\text { 2- Suporte de } \\
\text { Governamental }\end{array}$} & \multicolumn{6}{|c|}{ Quanto o Governo tem apoiado a sua empresa nas seguintes áreas, nos três últimos anos? } \\
\hline & CeG_1 & Vendas. & 0,34 & 0,80 & 0,51 & 0,20 \\
\hline & CeG_2 & Compras. & 0,38 & 0,85 & 0,53 & 0,26 \\
\hline & CeG_3 & Serviços financeiros (poupança, crédito, seguros, etc.). & 0,35 & 0,74 & 0,46 & 0,22 \\
\hline & CeG_4 & Benefícios fiscais & 0,35 & 0,70 & 0,41 & 0,20 \\
\hline & CeG_5 & Capacitação técnica. & 0,33 & 0,83 & 0,48 & 0,14 \\
\hline & CeG_6 & Capacitação gerencial. & 0,34 & 0,85 & 0,51 & 0,18 \\
\hline & CeG_7 & Serviços de informação. & 0,36 & 0,84 & 0,50 & 0,21 \\
\hline & CeG_8 & Serviços de contratação de pessoal. & 0,42 & 0,82 & 0,55 & 0,15 \\
\hline & CeG_9 & Assistência legal. & 0,42 & 0,84 & 0,58 & 0,16 \\
\hline \multirow{10}{*}{$\begin{array}{l}\text { 3- Suporte } \\
\text { Institucional }\end{array}$} & \multicolumn{6}{|c|}{$\begin{array}{l}\text { Quanto as instituições não governamentais têm apoiado sua empresa nas seguintes } \\
\text { áreas nos últimos três anos? }\end{array}$} \\
\hline & CeSi_1 & Vendas. & 0,42 & 0,53 & 0,87 & 0,17 \\
\hline & CeSi_2 & Compras. & 0,44 & 0,52 & 0,88 & 0,19 \\
\hline & CeSi_3 & Serviços financeiros (poupança, crédito, seguros, etc.). & 0,40 & 0,49 & 0,75 & 0,15 \\
\hline & CeSi_4 & Benefícios fiscais. & 0,39 & 0,52 & 0,74 & 0,18 \\
\hline & CeSi_5 & Capacitação técnica. & 0,40 & 0,54 & 0,88 & 0,15 \\
\hline & CeSi_6 & Capacitação gerencial. & 0,36 & 0,50 & 0,89 & 0,19 \\
\hline & CeSi_7 & Serviços de informação. & 0,42 & 0,52 & 0,87 & 0,16 \\
\hline & CeSi_8 & Serviços de contratação de pessoal. & 0,40 & 0,55 & 0,82 & 0,13 \\
\hline & CeSi_9 & Assistência legal. & 0,38 & 0,50 & 0,80 & 0,15 \\
\hline \multirow{10}{*}{$\begin{array}{l}\text { 4- Desempenho } \\
\text { Percebido }\end{array}$} & \multicolumn{6}{|c|}{$\begin{array}{l}\text { Quão satisfeito você está com desempenho da sua empresa em cada um dos } \\
\text { seguintes fatores... }\end{array}$} \\
\hline & $\mathrm{DeV}$ & Nivel de vendas. & $-0,02$ & $-0,05$ & $-0,05$ & 0,61 \\
\hline & DeCV & Crescimento em vendas. & 0,10 & 0,08 & 0,11 & 0,74 \\
\hline & DeFc & Fluxo de caixa. & 0,14 & 0,20 & 0,18 & 0,83 \\
\hline & $\mathrm{DeCi}$ & Rendimento sobre o capital dos investidores. & 0,20 & 0,24 & 0,22 & 0,88 \\
\hline & $\mathrm{DeMb}$ & Margem bruta. & 0,07 & 0,09 & 0,03 & 0,69 \\
\hline & DeLL & Lucro líquido. & 0,09 & 0,17 & 0,09 & 0,78 \\
\hline & DeLV & Lucro líquido com relação a vendas. & 0,04 & 0,13 & 0,04 & 0,71 \\
\hline & DeROI & Retorno sobre o investimento. & 0,18 & 0,25 & 0,21 & 0,83 \\
\hline & Capacidad & le para fazer crescer seu negócio, reinvestindo os ganhos no próprio negócio. & 0,19 & 0,20 & 0,16 & 0,78 \\
\hline
\end{tabular}

Fonte: Elaborada pelo autor com base nos dados coletados. 
TABELA 2: CORRELAÇÃO E ESTATÍSTICAS DESCRITIVAS DAS VARIÁVEIS LATENTES DE 1ª ORDEM

\begin{tabular}{|c|c|c|c|c|}
\hline Variáveis latentes de $1^{\circ}$ ordem & 1 & 2 & 3 & 4 \\
\hline 1- Desempenho Percebido & 0,61 & & & \\
\hline 2- Sup. Familiar e Social & 0,19 & 0,69 & & \\
\hline 3- Sup. Governamental & 0,25 & 0,45 & 0,81 & \\
\hline 4- Sup. Institucional & 0,20 & 0,48 & 0,62 & 0,83 \\
\hline Média & 0,31 & 0,54 & 0,72 & 0,42 \\
\hline Mediana & 0,22 & 0,48 & 0,72 & 0,42 \\
\hline Desvio Padrão & 0,20 & 0,13 & 0,13 & 0,59 \\
\hline Variância média extraída & 0,38 & 0,48 & 0,65 & 0,69 \\
\hline Confiabilidade composta & 0,93 & 0,91 & 0,94 & 0,95 \\
\hline
\end{tabular}

Fonte: Elaborada pelo autor com base nos dados coletados.

Nota $_{1}$ : Os valores em negrito, na diagonal, referem-se à raiz quadrada da variância média extraída.

Nota $_{2}$ : Escores fatoriais padronizados, variância média extraída e confiabilidade composta calculados por meio do software SmartPLS 2.0M3 (RINGLE; WENDE e WILL; 2005).

A validade discriminante foi identificada para amostra utilizada nesse estudo, uma vez que as cargas fatoriais encontradas entre as assertivas e seus respectivos constructos foram maiores do que aquelas encontradas nas relações com outros constructos. Por sua vez, a vali-dade convergente foi identificada para os constructos de suporte de governo e Suporte Insti-tucional, nos quais os índices foram superiores a $50 \%$. Entretanto, os índices de desempenho e suporte familiar e institucional apresentaram, respectivamente, a raiz quadrada da variância média extraída abaixo de $50 \%$, indicando falta de validade convergente para esses construc-tos. De acordo com Pichardo e Garza (2011) esse resultado aponta para uma limitação da amostra utilizada, todavia, como esse instrumento apresentou validade convergente em estu-dos anteriores, por exemplo, optou-se por apontar essa como uma limitação da amostra, e não um impeditivo para o estudo. Por último a validade composta foi confirmada, já que todos os índices foram superiores a 0,7, conforme o proposto por Chin (1998).
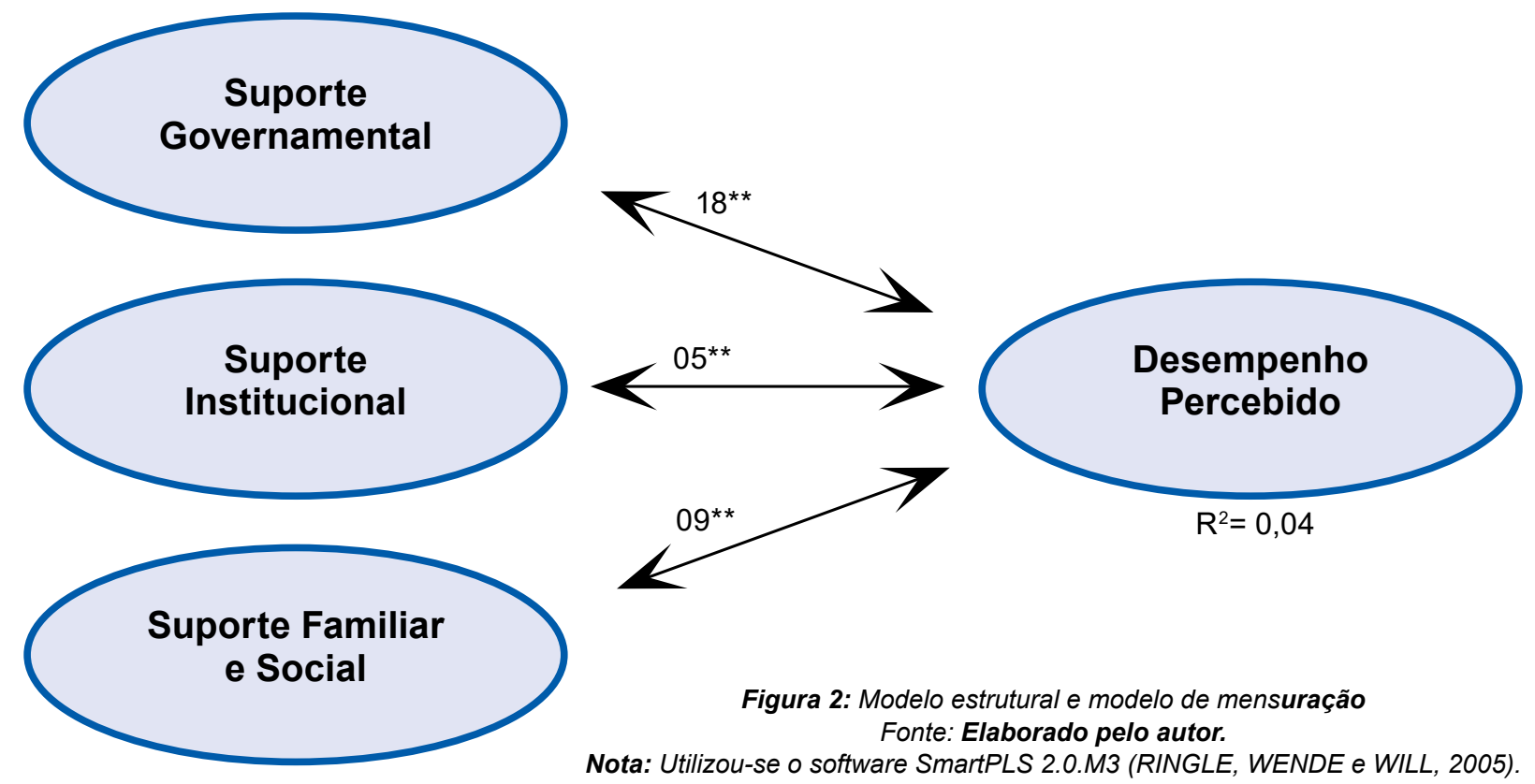
Fonte: Elaborado pelo autor.

Nota: Utilizou-se o software SmartPLS 2.0.M3 (RINGLE, WENDE e WILL, 2005). Significância estimada por meio de bootstrap com $N=217$ e 1000 repetições. Nota2: ${ }^{* *} p<0,01$ 
$\mathrm{O}$ modelo estrutural apresentado diz respeito às relações entre as variáveis latentes "Su-porte Governamental", "Suporte Institucional" e "Suporte Familiar e Social", e "Desempenho Percebido", todas de $1^{\mathrm{a}}$ ordem.

$\mathrm{O}$ coeficiente de caminhos dos constructos Suporte Governamental e Desempenho Per-cebido foi de 0,187 , indicando que há uma relação positiva entre eles, também comprovada entre os constructos Suporte Institucional e Desempenho Percebido onde o coeficiente foi de 0,05, e entre Suporte Familiar e Social e Desempenho Percebido cujo coeficiente foi de 0,09 .

\section{DISCUSSÃO DOS RESULTADOS}

Os resultados encontrados mostraram que o $\mathrm{Ca}$ pital Social composto pelos Suportes de Governo, Institucional, Familiar e Social, são constructos importantes e significativos ao expli-car o Desempenho Percebido, porém, sozinhos, mostram ser a explicação de um total de ape-nas 4,3\% do Desempenho Percebido nas MPEs relatados pelos participantes. Apesar de esse ser considerado um percentual pequeno de explicação, não se deve ignorar que a relação en-contrada foi significante e que, diante das multiplicidades de variáveis que podem explicar o desempenho, identificar uma variável não relacionada diretamente a fatores de competição como o Capital Social, por ser antecedente do desempenho, é algo relevante para a literatura.

De forma geral, os resultados sugerem que o $\mathrm{Su}$ porte Governamental foi, dentre os tipos de suporte estudados, o que gerou maior influência no Desempenho Percebido das MPEs es-tudadas. Este resultado acrescenta à literatura informações importantes, porquanto a grande maioria dos estudos prévios sobre a influência do Capital Social sobre o Desempenho o fize-ram sob uma ótica unidimensional, como notado nos trabalhos de Rowley e Berman (2000), Lee et al. (2001), e Park e Luo (2001). A diferença na dimensionalidade da mensuração do construto "Capital Social" pode ser uma explicação para o fato de nas referidas pesquisas não terem sido encontradas relações significantes entre esses constructos.

Em concordância, o estudo de Pichardo e Garza (2011) revelou resultados gerais seme-lhantes aos aqui encontrados, mesmo tendo sido retirado em outro contexto (México). A con-dição de país emergente compartilhada por ambas as economias pode ter sido mais um fator contextual colaborador para os resul- tados conseguidos nesta pesquisa e na de Pichardo e Gar-za (2011)

O Suporte Governamental em países em desenvolvimento é visto tradicionalmente como paternalista, com medidas politicas de curto prazo setoriais e individuais, em detrimento a ações estratégicas coletivas com resultados mais efetivos, porém no longo prazo (DENNIS JR, 2011). Politicas publicas como essas acabam indo ao encontro da eficiência coletiva proposta e preconizada pela Teoria das Redes Sociais (MESQUITA; LAZZARINI, 2008; SVETLIČIČ et al., 2007).

A forma mais comum em que o Suporte Governamental e Institucional são ofertados as MPEs em países em desenvolvimento é a forma indireta, ou seja, eles usam suas Redes Sociais para criar eventuais benefícios para as MPEs (KONTINEN; OJALA, 2011; PICHARDO et al., 2011; LIN, 1999), todavia, graças a dificuldade das MPEs fazerem a gestão da informação de forma estratégica, acabam percebendo pouco esses tipos de oportunidade, logo de suporte, para ampliar sua vantagem competitiva (POWELL, 1990, OLK, 2005).

Essa baixa percepção também foi evidenciada neste estudo, tornando assim dificultoso analisar o incremento desses tipos de suporte no Desempenho Percebido nas MPEs. Dessa forma, visando aumentar a relação positiva desses constructos no Desempenho Percebido, é sugerido que esses tipos de suporte sejam feitos de forma direta, diminuindo, por exemplo, a assimetria de informação, a burocracia e os custos para a obtenção de linhas de crédito para as MPEs, tal qual sugerido por Park e Luo (2001), Craig et al. (2008). Essa atuação direta pode mostrar ao empreendedor que não há impacto no aumento substanciais dos seus custos, em relação ao investimento em Capital Social por meio desses tipos de suportes, diferentemente, do que fora sugerido por Rowley e Berman (2000), Lee et al. (2001) e Park e Luo (2001).

Ao analisar a utilização das Redes Sociais pelas MPEs, parece que as empresas desse segmento possuem dificuldades de acessar o Capital Social - em suas três dimensões aqui apresentadas - e usufruir dele pelas suas redes sociais, diferentemente de como fazem as gran-des empresas (FAFCHAMPS; MINTEN, 2002). Essa comparação é relevante, pois pode cor-roborar com as descobertas feitas por Rowley e Berman (2000), Lee et al. (2001) e Park e Luo (2001), os quais afirmam que as MPEs consideram oneroso manter a formação de laços nas Redes Sociais. Dessa forma, as MPEs acabam por terem uma dificuldade 
de obter informa-ções relevantes e estratégicas para compor seu diferencial de mercado, gerando, assim, menos desempenho (POWELL, 1990; OLK, 2005; UZZI; GILLESPIE, 2002; WU, 2008).

Um outro ponto importante na discussão é a mensuração do Desempenho Percebido pe-las MPEs. Tanto neste estudo quanto no realizado no México por Pichardo e Garza (2011) optou-se por usar os parâmetros sugeridos por Covin e Slevin (1989), pois tais referências são comuns no dia a dia de uma MPE. Os modelos sugeridos como nível e crescimento das ven-das, margem bruta, lucro líquido em relação às vendas, etc, são, além de termos comuns, tam-bém são de fácil comparação com as práticas usadas na concorrência das MPEs. Dessa manei-ra, mesmo não havendo um banco de dados confiável ou a obrigatoriedade legal de publicação dos dados financeiros pelas MPEs, usando a percepção do empreendedor na sua rotina diária, é possível mensurar de forma positiva o Desempenho Percebido apresentado neste trabalho.

Como notado, a grande maioria dos respondentes concentrou-se no estado do Espírito Santo, o qual está em uma vertente de crescimento acima da média nacional, normalmente com a exportação de commodities - aço, minério de ferro, celulose, e agora, petróleo e gás - (CAÇADOR; GRASSI, 2011). Ainda segundo os autores, o Espírito Santo concentra 2\% do PIB Nacional, ocupa a $5^{\text {a }}$ colocação no PIB per capita, e atingiu o patamar de alto desenvol-vimento humano (IDH), ficando acima de 0,8 e mantém-se na $7^{\mathrm{a}}$ colocação entre os estados brasileiros nesse índice.

De forma geral, nota-se que o presente estudo colaborou com a literatura no sentido de apresentar uma mensuração robusta e multidimensional do Capital Social e sua relação com o Desempenho Percebido, como um todo. Os resultados sugerem um baixo, porém significante potencial de explicação do desempenho pelo Capital Social.

\section{CONCLUSÃO}

Esta pesquisa acaba auxiliando aos empreendedores gestores de MPEs a ampliarem e melhorarem suas relações por intermédio de suas Redes Sociais, especialmente, as informações cotidianas e fundamentais para a gestão do negócio, de forma cooperativa, pois elas tornam-se cruciais para o processo de aprendizagem empresarial. Contudo, os suportes aqui apresen- tados não devem ser priorizados como estratégicos, tampouco visto como fatores preponderantes no diferencial estratégico ou ainda como vantagem competitiva no negócio tendo em vista terem sido encontradas baixas relações entre os Suportes Governamental e Institucional com o De-sempenho Percebido.

Por outro lado, especificamente aqui é aberta uma gama de oportunidades, tanto para programas de $\mathrm{Su}-$ porte Governamental, quanto Suporte Institucional, para que possam atuar com mais assertividade com seus programas e ampliar seus canais de comunicação na forma direta, nas MPEs, que tem tido pouca percepção da responsabilidade desses indivíduos em contribuir de fato com o seu processo de desempenho dentro delas.

Vale destacar como medidas preventivas ligadas ao Suporte Familiar e Social que os gestores de MPEs - haja vista a participação efetiva de membros da família no cotidiano da empresa - invistam em capacitação técnicas de seus membros e também nos processos de su-cessão familiar.

O estudo apresenta como limitação o fato de a amostra ser de caráter transversal e não aleatório, o que impossibilita realizar afirmações causais entre os constructos e generalizar os resultados para todas as empresas componentes do universo de interesse do estudo. Outra li-mitação importante se refere ao fato já salientado de que o desempenho das MPEs estudados nessa pesquisa não foi medido a partir de indicadores objetivos, mas sim, por meio de respos-tas a uma escala de autopercepção.

Para pesquisas futuras, sugere-se ser o estudo realizado em um design longitudinal, de forma a verificar os efeitos da criação de Capital Social no desempenho ao longo do tempo. Além disso, sugere-se também que novos estudos utilizem dados financeiros das empresas estudadas para obter uma mensuração mais precisa do desempenho. Outra possibilidade seria coletar dados em diferentes contextos culturais de forma a buscar as variáveis ambientais e entender como podem influenciar os constructos propostos, por meio do Suporte Institucional, via Agências de Desenvolvimento do Empreendedorismo (ADE). Neste estudo foi avaliado o efeito direto dos três tipos de suporte no desempenho da MPE e, dessa forma, sugere-se, para pesquisas futuras, avaliar o papel de variáveis mediadoras, tais como tempo de existência e número de funcionários da empresa e o grau de competência empreendedora nessa relação. 
BOL, J.C.; SMITH, S.D. Spillover effects in subjective performance evaluation: bias and the asymmetric influence of controllability. The Accounting Review. v. 86, n. 4, p. 1213-1230, 2011.

BRASIL. Lei complementar n. 123, de 14 de dezembro de 2006. Institui o Estatuto Nacional da Microempresa e da Empresa de Pequeno Porte. Diário Oficial [da] República Federativa do Brasil. Brasília, 14 dez. 2006. Disponivel em: $<$ http://www.jusbrasil.com.br/diarios/466012/dou-edicao-extra-secao-1-31-01-2009-pg-1/pdf $>$. Acesso em: $01 \mathrm{dez} .2012$.

. Ministério do Trabalho e Emprego. Anuário dos trabalhadores. 2009. Disponível em: $<$ http:// www.dieese.org.br/anu/anuarioTrabalhadores2009/Arquivos/ANUARIO_TRABALHADORES_2009v.pdf>. Acesso em: 29 set. 2012.

BRASS, D. J. et al. Taking stock of networks and organizations: a multilevel perspective. Academy of Management Journal, v. 47, n. 6, p. 795-817, 2004.

BROCKMAN, B. K.; JONES, M. A.; BECHERER, R. C. Customer orientation and perfor-mance in small firms: examining the moderating influence of risk-taking, innovativeness, and opportunity focus. Journal of Small Business Management, v. 50, n. 3, p. 429-446, 2012.

BURT, R. S. The network structure of social capital. In: SUTTON, R. I.; STAW, B. M. (Eds.). Greenwich research in organizational behavior. Conn: JAI Press, 2000.

1997.

. The contingent value of social capital. Administrative Science Quartely, v. 42, n. 2. p. 339-365,

CAÇADOR, S. B.; GRASSI, R. A. A evolução recente da economia do Espírito Santo: um estado desenvolvido e periférico? In: ENCONTRO NACIONAL DE ECONOMIA (AN-PEC), 37., 2009, Foz do Iguaçu (PR). Anais... Foz do Iguaçu: ANPEC, 2009. Disponível em: <http://www.anpec.org.br/encontro2009/inscricao.on/ arquivos/000-acbb55edea8d55d858feb624d6b49f0d.pdf> Acesso em: 20 dez. 2012.

CHIN, W. W. The partial least squares approach to structural equation modeling. In: MAR-COULIDES, G. A. (ed.). Modern methods for business research. Mahwah: Lawrence Erl-baum Associates, Inc., 1998.

COVIN. J. G.; SLEVIN, D. P. Strategic management of small firms in hostile and benign environments. Strategic Management Journal, v. 10, n. 1, p. 75-87, 1989.

CRAIG, B. R.; JACKSON III, W. E.; THOMSON, J. B. Credit market failure interven-tion: do government sponsored small business credit programs enrich poorer areas? Small Bus Econ., v. 30, n. 4, p. 345-360, 2008.

DENNIS JR, W. J. Entrepreneurship small business and public policy levers. Journal of Small Business Management, v. 49, n. 2, p. 149-162, 2011.

ENGELLANDT, A.; RIPHAHN R. T. Evidence on incentive effects of subjective per-formance evaluations. Industrial and Labor Relations Review, v. 64, n. 2, p. 241-257, jan. 2011.

EUROPEAN COMISSION. European SMEs under pressure: annual report on EU small and mediumsized enterprises. 2009. Disponível em: <http://ec.europa.eu/enterprise/policies/sme/facts-figures-analysis/ performance-review/files/supporting-documents/2009/annual-report_en.pdf>. Acesso em: 15 set. 2012. 
FAFCHAMPS, M.; MINTEN, B. Social capital and the firm: evidence from agricultural trad-ers in Madagascar. In: GROOTAERT, C. H.; BASTELAER, T. (Eds.). The role of social capital in development: an empirical assessment. New York: Cambridge University Press, 2002. p. 125-152.

FILION, L. J. Empreendedorismo: empreendedores e proprietários-gerentes de pequenos ne-gócios. RAUSP - Revista de Administração da USP, São Paulo, v. 34, n. 2, p. 05-28, abr./jun. 1999.

. The Definition of Small Business as a Basic Element for Policy Making. Anais... "Small Business, Marketing and Society" (congresso). The Institute of Sociology, USSR Academy of Science, Academy of Science of Georgia, Centre for Sociology (orgs.). Tbilisy (Georgia - Rússia). 1991.

FITZGERALD, M. A. et al. Socially responsible processes of small family business owners: exploratory evidence from the national family business survey. Journal of Small Business Management, v. 48, n. 4, p. 524-551, 2010.

GELETKANYCZ, M. A.; HAMBRICK, D. C. The external ties of top executives: implica-tions for strategic choice and performance. Administrative Science Quarterly, v. 42, n. 4, p. 654-681, dec. 1997.

GILL, A. S. et al. Factors that influence financial leverage of small business firms in India. Intemational Joumal of Economics and Finance, v. 4, n. 3, p. 33-45, mar. 2012.

GILL, A. The impact of board size and CEO duality on the potential growth of Canadian Manufacturing firms. In: INTERNATIONAL ACCOUNTING CONFERENCE (IAC), 2011, Surabaya (IND). Anais... Surabaya: IAC, 2011.

GOLDMARK, L. Microenterprise development in Latin America: towards a new flexibility. Journal of Socio-Economics, v. 30, n. 2, p. 145-149, 2001.

HAIR JR., J. F. et al. Análise multivariada de dados. 5. ed. Porto Alegre: Bookman, 2005. . et al. Multivariate data analysis. Englewood Cliffs, NJ: Prentice-Hall, 1998.

HUDSON, C. W. et al. Tradução, adaptação cultural e análise de consistência interna do in-ventário de externalização. Aval. psicol., Porto Alegre, v. 6, n. 2, p. 217-227, dez. 2007.

JÄNKÄLÄ, S.; SILVOLA, H..Lagging effects of the use of activity-based costing on the financial performance of small firms. Journal of Small Business Management, v. 50, n. 3, p. 498-523, 2012.

KONTINEN, T.; OJALA, A. Social capital in relation to the foreign market entry and post-entry operations of family SMEs. J. Int. Entrep., v. 9, n. 2, p. 133-151, 2011.

KOTANE, I.; MERLINO, I. K.. Assessment of financial indicators for evaluation of business performance. European Integration Studies, n. 6, p. 216-224, 2012.

LEE, C., LEE, K.; PENNINGS, J. M. Internal capabilities, external networks, and perfor-mance: a study on technology-based ventures. Strategic Management Journal, v. 22, n.6, p. 615-40, 2001.

LI, Y. et al. Moderating effects of entrepreneurial orientation on market orientation-performance linkage: evidence from chinese small firms. Journal of Small Business Management, v. 46, n. 1, p. 113-133, 2008.

LIN, N. Building a network theory of social capital. Connections, v. 22, n. 1, p. 28-51, 1999. 
MACAU, F. R.; BRITO, L. A. L. Knowledge effects on performance in manufacturing and service firms. In: ENCONTRO DA ASSOCIAÇÃO DOS PROGRAMAS DE PÓS GRA-DUAÇÃO E PESQUISA EM ADMINISTRAÇÃO (ENANPAD), 34., 2010, Rio de Janeiro (RJ). Anais... Rio de Janeiro: ANPAD, 2010.

MACIEL, C. O.; CAMARGO, C. Lócus de controle, comportamento empreendedor e de-sempenho de pequenas empresas. RAM - Revista de Administração Mackenzie, São Paulo, v. 11, n. 2, p. 168-188, mar./ abr. 2010.

MARTELETO, R. M. Análise de redes sociais: aplicação nos estudos de transferência de in-formação. Ciência da Informação, Brasília, v. 30, n. 1, p. 71-81, 2001.

MESQUITA, L. F.; LAZZARINI, S. G. Horizontal and vertical relationships in developing economies: implications for SMEs access to global markets. Academy of Management Journal, v. 51, n. 2, p. 359-380, 2008.

MIKHAILITCHENKO, A. G. Symbiotic networks in SME internationalization: a U.S.-China-Russia study. 2008. 237 f. Tese (Doutorado em Administração) - Department of Mar-keting at the College Graduate, Cleveland State University, Cleveland (USA), 2008.

MOHAMAD, O. et al. The role of business environment as a moderator. The IUP Journal of Management Research, v. 10, n. 3, p. 7-27, 2011.

NAHAPIET, J.; GOSHAL, S. Social capital, intellectual capital, and the organizational ad-vantage. Academy of Management Review, v. 23, n. 2, p. 242-266, 1998.

OLK, P. Modeling and measuring the performance of alliances. In: SHENKAR, O.; REUER, J.J. (Eds.). Handbook of strategic alliances. London: Sage, 2005.

PARK, S. H.; LUO, Y. Guanxi and organizational dynamics: organizational networking in Chinese firm. Strategic Management Journal, v. 22, n. 5, p. 455-77, 2001.

PETARAF, M. A.; BARNEY, J. B. Unraveling the resource-based tangle. Managerial and Decision Economics, v. 24, n. 4, p. 309-323, 2003.

PETT, M. A.; LACKEY, N. R.; SULLIVAN, J. Making sense of factor analysis: the use of factor analysis for instrument development in health care research. California: SAGE, 2003.

PICHARDO, R. D.; ARRIAGA, J. M.; COBAS, E. F. Improving entrepreneurial competency in low-income segments: the impact of entrepreneurial development agencies. In: WORLD CONFERENCE BACK TO THE FUTURE: CHANGES IN PERSPECTIVES OF GLOBAL ENTREPRENEURSHIP AND INNOVATION, INTERNATIONAL COUNCIL FOR SMALL BUSINESS, 2011, Stockholm (SWE). Anais... Stockholm: ICSB, 2011.

POWELL, W. W. Neither market nor hierarchy: Network forms of organization. Research in organizational Behavior, v. 12, p. 295-336, 1990.

PRAHALAD, C.K.; HART, S. The fortune at the bottom of the pyramid. Strategy + Business, v. 26, p.1-14, jan. 2002.

PROVAN, K. G.; SYDOW, J. Evaluating inter-organizational relationships. In: CROPPER, S. et al. The Oxford handbook of inter-organizational relations. Oxford: Oxford Universi-ty Press, 2008. 
QUATMAN, C.; CHELLADURI, P. Social network theory and analysis: a complementary lens for inquiry. Journal of Sport Management, v. 22, n. 3, p. 338-360, 2008.

RINGLE, C. M.; WENDE, S.; WILL, A. Smart PLS 2.0 M3 (beta). Germany: University of Hamburg, 2005. ROWLEY, T.; BERMAN, S. A. Brand new brand of corporate social performance. Business and Society, v. 39, n. 4, p. 397-418, 2000.

SEBRAE. Serviço Brasileiro de Apoio às Micro e Pequenas Empresas. Taxa de sobrevivência das empresas no Brasil. Brasília: UGE, 2011. (Coleção Estudos e Pesquisas). Disponível em: $<$ http://www.biblioteca.sebrae. com.br/bds/bds.nsf/45465B1C66A6772D832579300051816C/\$File/NT00046582.pdf>. Acesso em: 15 jul. 2012.

SVETLIČIČ, M.; JAKLIC, A.; BURGER, A. Internationalization of small and medium-size enterprises from selected Central European economies. Eastern European Economics, v. 45, n. 4, p. 36-65, 2007.

SYDOW, J.; MILWARD, H. B. Reviewing the evaluation perspective: on criteria, occasions, procedures and practices: Special Interest Group (SIG) on Interorganizational Relations (IOR). In: International Conference on Multi-Organisational Partnerships, Alliances and Net-works (MOPAN), 10., Glasgow (ESC). Anais... Glasgow: MOPAN, 2003.

TEECE, D. Firm capabilities and economic development: implications for the newly industri-alizing economies. In: KIM, L.; NELSON, R. (Eds.). Technology, learning \& innovation. Cambridge: University Press, 2000.

TERZIOVSK, M. Innovation practice and its performance implications in small and medium enterprises (SMEs) in the manufacturing sector: a resource-based view. Strategic Management Journal, v. 31, p. 892$902,2010$.

TODOROVA, G.; DURISIN, B. Absorptive capacity: valuing a reconceptualization. Academy of Management Review, v. 32, n. 3, p. 774-786, 2007.

TRIGUEROI, R. et al. Human resource management practices aimed at seeking the commitment of employees on financial and non-financial (subjective) performance in spanish firms: an empirical contribution. Journal of Economics, Finance and Administrative, v. 17, n. 32, jun. 2012

UZZI, B.; GILLESPIE, J. J. Knowledge spillover in corporate financing networks: embeddedness and the firm's debt performance. Strategic Management Journal, v. 23, p. 595-618, 2002.

VAN WIJK, R.; JANSEN, J. J.; LYLES, M. A. Inter- and Intra-organizational knowledge transfer: a metaanalytic review and assessment of its antecedents and consequences. Journal of Management Studies, v. 45, p. 830-853, 2008.

WASSERMAN, S.; FAUST, K. Social network analysis: methods and applications. Cambridge: University Press, 1994.

WESTLUND, H; ADAM, F. Social capital and economic performance: a meta-analysis of 65 studies. European Planning Studies. v. 18, n. 6, p. 893-919, 2010.

WIER, B.; HUNTON, J.; HASSAB, H. R. Enterprise resource planning systems and non-financial performance incentives: the joint impact on corporate performance. International Journal of Accounting Information Systems, v. 8, n. 3, p. 165-190, 2007. 
WOOLCOCK, M. Microenterprise and social capital: a framework for theory, research, and policy. Journal of Socio-Economics, v. 30, n. 2, p. 193-198, 2001.

WRIGHT, M.; FILATOTCHEV, I.; HOSKISSON, R. E.; PENG, M. W. Strategy research in emerging economies: challenging the conventional wisdom. Journal of Management Studies, v. 42, n. 1, p. 1-33, 2005.

WU, W. P. Dimensions of social capital and firm competitiveness improvement: the mediating role of information sharing. Journal of Management Studies, v. 45, n. 1, p. 122-146, 2008.

YANG, Y.; YU, W.Y. Effect of board governance on corporate growth-based on empirical data of chinese a-share top 500 listed companies. Journal of Xidian University, Social Sci-ence Edition, n. 3, jul. 2011. Disponível em: <http://en.cnki.com.cn/Article_en/CJFDTOTAL-XADZ201103003.htm>.

YIU, D. W.; LAU, C. M. Corporate entrepreneurship as resource capital configuration in emerging market firms. Entrepreneurship: Theory and Practice, v. 32, n. 1, p. 37-57, 2008.

ZAHER, A.; BELL, G. G. Benefiting from network position: firm capabilities, structural holes and performance. Strategic Management Journal, v. 26, p. 809-825, 2005.

ZAHRA, S. A.; UCBASARAN, D.; NEWEY, L. R. Social knowledge and SMEs' innovative gains from internationalization. European Management Review, v. 6, p. 81-93, 2009.

ZHAO, H.; HSU, C. Social ties and foreign market entry: an empirical inquiry. Management International Review, v. 47, n. 6, p. 815-844, 2007. 